\title{
PHASE RELATIONSHIP BETWEEN SKIN TEMPERATURE AND SLEEP- WAKE RHYTHMS IN WOMEN WITH VASCULAR DYSREGULATION AND CONTROLS UNDER REAL-LIFE CONDITIONS
}

\author{
Britta Gompper, ${ }^{1}$ Vivien Bromundt, ${ }^{1}$ Selim Orgül, ${ }^{2}$ Josef Flammer, ${ }^{2}$ and \\ Kurt Kräuchi, ${ }^{1}$ \\ ${ }^{1}$ Thermophysiological Chronobiology, Centre for Chronobiology, \\ Psychiatric Hospital of the University of Basel, Switzerland \\ ${ }^{2}$ University Eye Clinic, Basel, Switzerland
}

The aim of the study was to investigate whether women with primary vascular dysregulation (VD; main symptoms of thermal discomfort with cold extremities) and difficulties initiating sleep (DIS) exhibit a disturbed phase of entrainment $(\Psi)$ under everyday life conditions. The authors predicted a phase delay of the distal-proximal skin temperature gradient and salivary melatonin rhythms with respect to the sleepwake cycle in women with VD and DIS (WVD) compared to controls (CON), similar to that found in their previous constant-routine laboratory data. A total of 41 young healthy women, 20 with WVD and 21 matched CON without VD and normal sleep onset latency (SOL), were investigated under ambulatory conditions (following their habitual bedtimes) during 7 days of continuous recording of skin temperatures, sleepwake cycles monitored by actimetry and sleep-wake diaries, and single evening saliva collections for determining the circadian marker of dim light melatonin onset (DLMO). Compared to CON, WVD showed increased distal vasoconstriction at midday and in the evening, as indicated by lower distal (DIST; hands and feet) and foot-calf skin temperatures, and distal-proximal skin temperature gradients $(p<.05)$. WVD manifested distal vasoconstriction before lights-off that also lasted longer after lights-off than in CON. In parallel, WVD exhibited a longer SOL $(p<.05)$. To define internal phase-relationships, cross-correlation analyses were performed using diurnal rhythms of wrist activity and foot skin temperature. WVD showed a phase delay in foot skin temperature (CON versus WVD: $3.57 \pm 17.28 \mathrm{~min}$ versus $38.50 \pm$ $16.65 \mathrm{~min} ; p<.05)$ but not in wrist activity. This finding was validated by additional within-subject cross-correlation analyses using the diurnal wrist activity pattern as reference. DLMO and habitual sleep times did not differ between CON and WVD. The authors conclude that WVD exhibit a phase delay of distal vasodilatation with respect to their habitual sleep-wake cycle and other circadian phase markers, such as DLMO. A full factorial design will have to show whether the finding is specific to

Submitted May 3, 2010, Returned for revision June 4, 2010, Accepted August 31, 2010

Address correspondence to Kurt Kräuchi, Thermophysiological Chronobiology, Centre for Chronobiology, Psychiatric Hospital of the University of Basel, Wilhelm Klein Strasse 27, 4012 Basel, Switzerland. Tel: ++ 4161325 5508; Fax: ++ 4161325 5556; Email: Kurt.kraeuchi@upkbs.ch 
primary vascular dysregualtion, to DIS, or to their interaction. (Author correspondence: Kurt.kraeuchi@upkbs.ch)

Keywords Ambulatory measurements; Circadian rhythm thermoregulation; Melatonin; Skin temperatures; Difficulties initiating sleep; Vascular dysregulation

\section{INTRODUCTION}

Under real-life conditions, the endogenous circadian pacemaker in the suprachiasmatic nuclei (SCN) is precisely entrained to the 24-h solar day. Each overt rhythm is characterized by a certain "phase of entrainment" $(\Psi)$, either with respect to other circadian rhythms (= internal $\Psi$ ) or with respect to the zeitgeber, e.g., light-dark cycle (= external $\Psi$ ). Both internal and external $\Psi$ are characteristics of the organism's entrained state (Roenneberg et al., 2003a). The two most extensively used marker rhythms in human circadian studies are core body temperature (CBT) and salivary or plasma melatonin. Important for a stable CBT is that body heat production and heat loss be in balance (Aschoff, 1971; Werner, 2009). The distal skin regions (mainly hands and feet) play a crucial role in the regulation of the endogenous circadian CBT rhythm (Aschoff \& Heise, 1972).

The endogenous circadian time course of distal skin temperatures (hands and feet) exhibits an inverse and higher amplitude rhythm than CBT when measured during the demasking conditions of a constantroutine protocol (CR) (Kräuchi \& Wirz-Justice, 1994; Kräuchi et al., 2006). In contrast, temperatures of proximal skin regions, e.g., thigh, infraclavicular region, stomach, forehead, passively follow CBT with similar amplitude (Aschoff \& Heise, 1972; Kräuchi \& Wirz-Justice, 1994; Kräuchi et al., 2006). This opposite circadian rhythm in distal and proximal skin temperature rhythms reflects the differences in thermophysiological regulatory mechanisms.

Heat loss via distal skin regions is essentially promoted by opening of arteriovenous anastomoses (AVAs), exclusively found in these glabrous skin regions. AVAs are shunts between arterioles and venules, and when they are open blood streams rapidly from the core to the distal skin regions, leading to efficient body heat loss and subsequent decrease in CBT (Hales, 1985). The distal-proximal skin temperature gradient (DPG) provides a selective measure of distal skin blood flow, and, hence, of efficient body heat loss via the extremities (House \& Tipton, 2002; Rubinstein \& Sessler, 1990; Severens et al., 2010). It has previously been shown that the increase in distal skin temperature and decline in CBT in the evening is associated with melatonin secretion onset, subjective sleepiness, and ease of falling asleep (Campbell \& Broughton, 1994; Kräuchi et al., 2007; Lack et al., 2008). 
Part of the general population, mostly women before menopause, exhibit a primary vasospastic dysregulation (VD) (Flammer et al., 2001). These individuals appear to maintain a large thermophysiological shell (Aschoff, 1971) with constricted vessels in distal skin regions to protect their core from cooling. Women with VD have a diathesis of responding with spasm to stimuli like cold temperature or emotional stress, particularly concerning the hands and feet. Additionally, they often suffer from difficulties initiating sleep (DIS). It is a prevalent symptom of high clinical relevance. In a recent epidemiological survey, we found that nearly $30 \%$ of women between the age of 20 and 40 yrs suffer from VD, but only $7 \%$ of men. In both sexes, the relative risk for DIS was doubled in subjects with VD (Kräuchi et al., 2008). In addition, we have shown that, under constant-routine laboratory conditions, women with both VD and DIS (WVD) exhibit a phase delay of the thermoregulatory system with respect to their sleep-wake rhythm (changed internal $\Psi$ ). As a consequence, their distal skin temperature is still low before lights-off, suggesting that they are not ready thermophysiologically to go to sleep, and this goes together with their observed prolonged sleep onset latency (Vollenweider et al., 2008). However, there is no information how WVD are entrained in their normal everyday life. WVD can be considered a "naturalistic model"these are healthy subjects, not sleep insomniacs-to study the relationship between sleep induction and thermoregulation. In this study, we specifically tested the hypothesis whether WVD exhibit a phase delay of distal skin temperatures with respect to their sleep-wake cycle under normal life circumstances, i.e., whether they have lower distal skin temperatures in the evening before lights-off than controls $(\mathrm{CON})$.

\section{METHODS}

\section{Participants}

Subjects were recruited by poster and Internet advertisements at the Universities of Basel (Switzerland), Freiburg i. Breisgau, Tübingen, and Mainz (Germany). A total of 20 healthy WVD (women with both VD and DIS) and 21 healthy CON (women without VD and DIS) were selected by means of screening questionnaires.

Two questions referring to the leading symptoms of VD were used for its definition:

1. During the past month, how intensively did you suffer from cold hands?

2. During the past month, how intensively did you suffer from cold feet? 
The answers were assessed on a Likert-scale: "not at all" =1, "a little" $=2$, "quite" $=3$, "extraordinary" $=4$. The criterion for VD was fulfilled if the subject answered both questions with either "quite" or "extraordinary" $(>2)$. CON had to answer both questions with "not at all" or "a little" $(<3)$. These criteria have been independently validated by finger skin temperature measurements after a cold stress (Kräuchi et al., 2008).

Three questions referring to the leading symptoms of DIS were used for its definition:

1. During the past month, how often was your sleep onset latency longer than 30 minutes? Answer categories: "never" =1, "seldom" = 2, "1-2 times per week" $=3, " \geq 3$ times per week" $=4$.

2. During the past month, was it a problem for you to fall asleep? Answer categories: "not at all" =1, "a little" = 2, "quite" $=3$, "extraordinary" $=4$.

3. How long did you take to fall asleep? Answer categories: $<5 \mathrm{~min}=1$, $5-14 \min =2,15-24 \min =3,>25 \min =4$.

DIS was rated when the sum score of all three questions was $>6$ and questions 1 and 2 were answered with $>2$. No DIS was rated when the sum score was $<6$ and all three answer categories were $<3$.

Furthermore, subjects were asked in screening questionnaires about their health ("How is your current health condition?"), and three questions addressed stress factors ("How often did you have stress in the past four weeks?"; "Do you get cold hands due to stress?"; and "Do you get warm hands due to stress?"). Answer categories are described in the legend to Table 1 .

Excluded were subjects with acute and chronic mental and physical diseases, nickel allergy, body mass index $<17.5$, migraine, smoking, medication or drug consumption, shiftwork within 3 months or transmeridian travel within 1 month before the study, excessive caffeine ( $\leq 2$ cups/day) or alcohol consumption ( $\leq 1$ glass/day). Other exclusion criteria were reports of disturbances in the sleep-wake cycle: delayed sleep phase syndrome (DSPS), advanced sleep phase syndrome, or sleep duration $<6$ or $>9 \mathrm{~h}$.

In women without hormone supplementation, data sampling took place during the luteal phase, defined as the interval between day 14 and the end of the menstrual cycle. Eleven CON and $10 \mathrm{WVD}$ were taking contraceptives. Based on the fact that no significant effects of contraceptives on the output variables (skin temperatures, sleep habits, or wrist activity) were found, the factor "contraceptives" was not taken into account (data not shown).

The study was conducted between the end of winter and beginning of summer (number of participants: $\mathrm{N}=8$ [February], 12 [March], 11 [April], 10 [May], 1 [June 2006]; the distribution of WVD and CON did 
TABLE 1 Biological and sleep parameters (subjective characteristics)

\begin{tabular}{lcrr}
\hline Variable & CON & WVD & $P$-value \\
\hline Age (years) & $25.00 \pm 1.00$ & $25.80 \pm 1.00$ & .56 \\
BMI $\left(\mathrm{kg} / \mathrm{m}^{2}\right.$ ) & $21.60 \pm .50$ & $20.40 \pm .40$ & .07 \\
Health (Likert scale) & $1.43 \pm .11$ & $1.95 \pm .15$ & $.008^{*}$ \\
Stress (Likert scale) & $2.52 \pm .19$ & $1.80 \pm 1.56$ & $.005^{*}$ \\
Cold hands due to stress (Likert scale) & $3.38 \pm .19$ & $2.32 \pm .20$ & $.004^{*}$ \\
Warm hands due to stress (Likert scale) & $3.57 \pm .15$ & $3.17 \pm .19$ & .09 \\
Time in bed before lights off (min) & $14.60 \pm 1.40$ & $27.00 \pm 2.30$ & $.03^{*}$ \\
Lights off (h) & $23.90 \pm .30$ & $24.00 \pm .20$ & .63 \\
Sleep latency (min) & $7.80 \pm 1.10$ & $29.20 \pm 4.30$ & $<.001^{*}$ \\
Sleep midpoint (h) & $3.90 \pm .20$ & $4.20 \pm .20$ & .30 \\
Sleep duration (h) & $7.70 \pm .20$ & $7.40 \pm .20$ & .26 \\
Lights on (h) & $7.70 \pm .20$ & $7.80 \pm .30$ & .65 \\
Time in bed after lights on (min) & $24.60 \pm 2.70$ & $39.10 \pm 7.20$ & .06 \\
Waking during night (number) & $.20 \pm .04$ & $0.40 \pm .07$ & $.005^{*}$ \\
Getting up during night (number) & $.06 \pm .02$ & $0.08 \pm .02$ & .5 \\
Sleep quality (Likert scale) & $5.80 \pm .30$ & $5.00 \pm .20$ & .05 \\
Restorative sleep (Likert scale) & $5.60 \pm .30$ & $5.00 \pm .20$ & .11 \\
Time outdoors (h) & $33.57 \pm 5.11$ & $21.11 \pm 4.39$ & .68 \\
\hline
\end{tabular}

Note. Values are means \pm SEM over 1 week. $p$ values are performed by Mann-Whitney $U$ test and one-way ANOVA. Age, body mass index $(\mathrm{BMI})$, health $(1=$ very good, $2=\operatorname{good}, 3=$ moderate, $4=$ bad, $5=$ very bad $)$, and stress factors $(1=$ frequent, $2=$ sometimes, $3=$ infrequent, $4=$ never $)$ were determined by screening questionnaires. Other variables were collected by the use of sleep-wake diaries. Sleep quality and restorative sleep were also measured by Likert scales $(1=$ very poor; $8=$ very good $)$.

not differ in a statistically significantly manner). Each subject signed an informed consent form approved by the local Ethical Committee (Ethikkommission beider Basel) for research on human subjects. The study conformed to the Declaration of Helsinki and met the ethical standards of the journal (Portaluppi et al., 2008). All subjects completed the study without any complaints.

\section{Skin Temperature Recordings}

Skin temperatures were recorded during a 1-wk ambulatory protocol under habitual life conditions. Wireless temperature sensors (DS 1922L Thermochron iButtons ${ }^{\circledR}$; diameter $\times$ height: $17 \times 6 \mathrm{~mm}$, accuracy $0.0625^{\circ} \mathrm{C}$; Maxim, Dallas, USA) were used to record skin temperatures continuously in 2.5-min intervals (Smith et al., 2010). The iButtons ${ }^{\circledR}$ were fixed to the skin with thin, air-permeable adhesive surgical tape (Fixomull $^{\circledR}$; Beiersdorf, Hamburg, Germany) on the left and right side of the body: foot (on the inner calcaneus bone), wrist (palm side of the wrist on the os lunatum), calf, thigh, and infraclavicular region, and one temperature sensor on the sternum (Van Marken Lichtenbelt et al., 2006). The temperature sensors can be worn under normal life conditions, including when taking a shower and participating in sports. Temperature 
data with their time stamps were then transferred to the statistic program (see below). "Proximal skin temperature" is defined as averaged skin temperatures of thigh, infraclavicular regions, and sternum. The mean skin temperatures of the hands and feet constitute the "distal skin temperature." All other skin measurements are calculated as the mean of the left and right side of the body.

\section{Actimetric Recordings}

Actimetry was used to monitor motor activity using Actiwatch ${ }^{\circledR}-\mathrm{L}$ devices (Cambridge Neurotechnology, UK). Wrist activity of the nondominant arm was recorded and stored in 1-min intervals, and daily sleep-wake logs were filled in by the subjects upon waking. Activity data were edited so that gaps due to actigraph removal, e.g., when showering, were filled with activity counts from the 24-h-day average. If $>3 \mathrm{~h} /$ day or $1 \mathrm{~h} /$ night of data were missing, then the data of the whole day were excluded from further analysis. Sleep parameters were calculated for 18 $\mathrm{CON}$ and $20 \mathrm{WVD}$ by the Sleep and Activity Analysis Software 7.23V (Cambridge Neurotechnology). The Non-Parametric Circadian Rhythm Analysis (NPCRA; Van Someren et al., 1999), calculated the relative amplitude of the 10-h of highest activity compared with the $5 \mathrm{~h}$ of least activity as a comprehensive representation of the amplitude of the sleepwake cycles $(\mathrm{CON} \mathrm{N}=18$; WVD $\mathrm{N}=19)$.

\section{Saliva Sampling for Melatonin Determination}

For the determination of the dim light melatonin onset (DLMO), nine saliva samples were collected on one evening under dim light conditions (subjects had to wear sunglasses) using Salivettes ${ }^{\circledR}$ (Sarstedt, Nümbrecht, Germany). Samples were taken at 30-min intervals starting $4 \mathrm{~h}$ before bedtime, and subjects were instructed to stay at home and avoid high activity this evening. Melatonin was assayed by a validated radioimmunoassay (Bühlmann Laboratories, Allschwil, Switzerland (Weber et al., 1997). DLMO was determined by linear interpolation of the evening melatonin rise across a $3 \mathrm{pg} / \mathrm{mL}$ threshold (Pandi-Perumal et al., 2007). Three subjects collected melatonin samples after the ambulatory week, but under similar life conditions. The data of two subjects were excluded from statistical analyses due to lack of compliance.

\section{Sleep-Wake Diaries}

Subjects rated their sleep episode directly in their sleep-wake diaries, immediately after awakening. The items were bedtime, lights-off, estimated sleep onset latency, sleep disturbances (waking bouts, get-up times 
during night), wake-up time (lights-on), and get-up time in the morning. Additionally, they rated their sleep quality, restorative sleep, and sleepiness before lights-off. The wake diary was divided into four time spans of the day: morning (06:00-12:00 h), afternoon (12:00-18:00 h), evening (18:00 h-sleep time), and shortly before lights-off. Estimations were made on $100 \mathrm{~mm}$ bipolar visual analogue scales (VAS): alert-sleepy, relaxed-tense, concentrated-unfocused, satiated-hungry, and good mood-bad mood (e.g., $0 \mathrm{~mm}=$ extremely alert, $100 \mathrm{~mm}=$ extremely sleepy). Additionally, self-ratings on bipolar VAS for thermal comfort were estimated for different skin regions: feet, body, and hands $(0 \mathrm{~mm}=$ feeling extremely cold, $100 \mathrm{~mm}=$ feeling extremely hot). Subjects noted timing of activity, time spent outdoors, and time of stressful psychological situations.

\section{Statistical Analysis}

The statistical packages StatView 5.0 (Abacus Concepts, Berkley, CA) and Statistica 7 for Windows (StatSoft, Tulsa, OK) were used. Statistical analyses were based on weekly averaged daily profiles per subject. In order to reduce short-term fluctuations and the number of time segments, continuously recorded data were averaged in 30-min blocks. The time courses were statistically analyzed by two-way, repeated-measurements analysis of variance (ANOVA). Huynh-Feldt (H-F) statistics were used to adjust the covariance matrix for violations of sphericity. H-F's $p$ values were based on corrected degrees of freedom, but the original degrees of freedom are reported. When the $F$ ratio proved significant, Curran-Everret, multiple-range, post hoc tests were applied to locate significant differences between means (Curran-Everett, 2000). Results are reported in detail when the significance level was $p<.05$. Simple-group differences between WVD and CON were calculated by factorial oneway ANOVA. Intraindividual phase relationships between feet skin temperatures and wrist activity were determined by cross-correlation analyses, using time series over 7 days of 5-min binned data (see Kräuchi \& Wirz-Justice, 1994; Kräuchi et al., 2006). Additionally, interindividual phase relationships between feet skin temperatures and wrist activity were analyzed for WVD and CON. Mean CON values $(\mathrm{N}=21)$ were taken as the reference curve for each subject. Time lags of maximum and minimum r-values were extracted from smoothed (by 65min moving averaged) individual curves between time lags of $\pm 960 \mathrm{~min}$. Mean cross-correlation curves were calculated after Fisher $z$-transformation of $r$ values, which were retransformed for Figure 3. The relation between the two variables was statistically tested by least-squares correlation analysis. 


\section{RESULTS}

\section{Subjects' Characteristics}

Table 1 summarizes the biological and sleep behavioral characteristics of the study subjects. Age and body mass index were similar between groups. All 41 subjects were in good health, though WVD were slightly worse than CON. WVD exhibited a higher level of stress and higher occurrence of cold hands due to stress. With respect to sleep characteristics, WVD reported significantly more awakenings during the night, longer time in bed before lights-off, and longer sleep onset latency. Subjectively estimated and objectively measured sleep latencies (by wristactimetry) were significantly correlated $(p<.05)$.

\section{Physiological Data Analyses}

In a first step, the daily time course of skin temperatures and wrist activity of WVD and CON were analyzed according to clock time (solar time), neglecting possible differences in intra- and interindividual sleepwake patterns, which was taken into account in a second step of analysis with data adjustment to the individual sleep-wake cycle (percentilized data for sleep and wake phases, separately). This kind of analysis has the disadvantage of expansion or contraction of the time axis, but gives a first impression of phase of entrainment with respect to the sleep-wake cycle. Thirdly, the phase relationships between the variables were calculated by cross-correlation analyses.

\section{Daily Time Course of Clock Time (Adjusted Data)}

Figures 1 and 2 (left panel) compare the daily patterns of ambulatory skin temperatures and wrist activity of WVD and CON with respect to clock time. Table 2 summarizes the findings of the repeated-measures ANOVA of all variables. All skin temperature measures show maximal values during the night and minimal values during the light phase. An additional peak was observed around 16:00 h. In general, only distal skin regions, DPG, and the foot-calf skin temperature gradient reveal significant differences between WVD and CON (interaction: Time $\times$ Group; significant differences between WVD and CON at a certain timepoints are indicated by $\left.{ }^{*} p<.05\right)$. In comparison to CON, WVD exhibit reduced values between 12:00 and 15:00 $\mathrm{h}$ and between 21:00 and 23:30 $\mathrm{h}$ (or until $00: 30 \mathrm{~h}$ for the foot-calf skin temperature gradient). In addition, lower 24-h mean values of the DPG and foot-calf skin temperature gradient are found in WVD. The 24-h mean levels of the distal skin temperatures are significantly lower than the proximal skin temperatures $(p<$ 

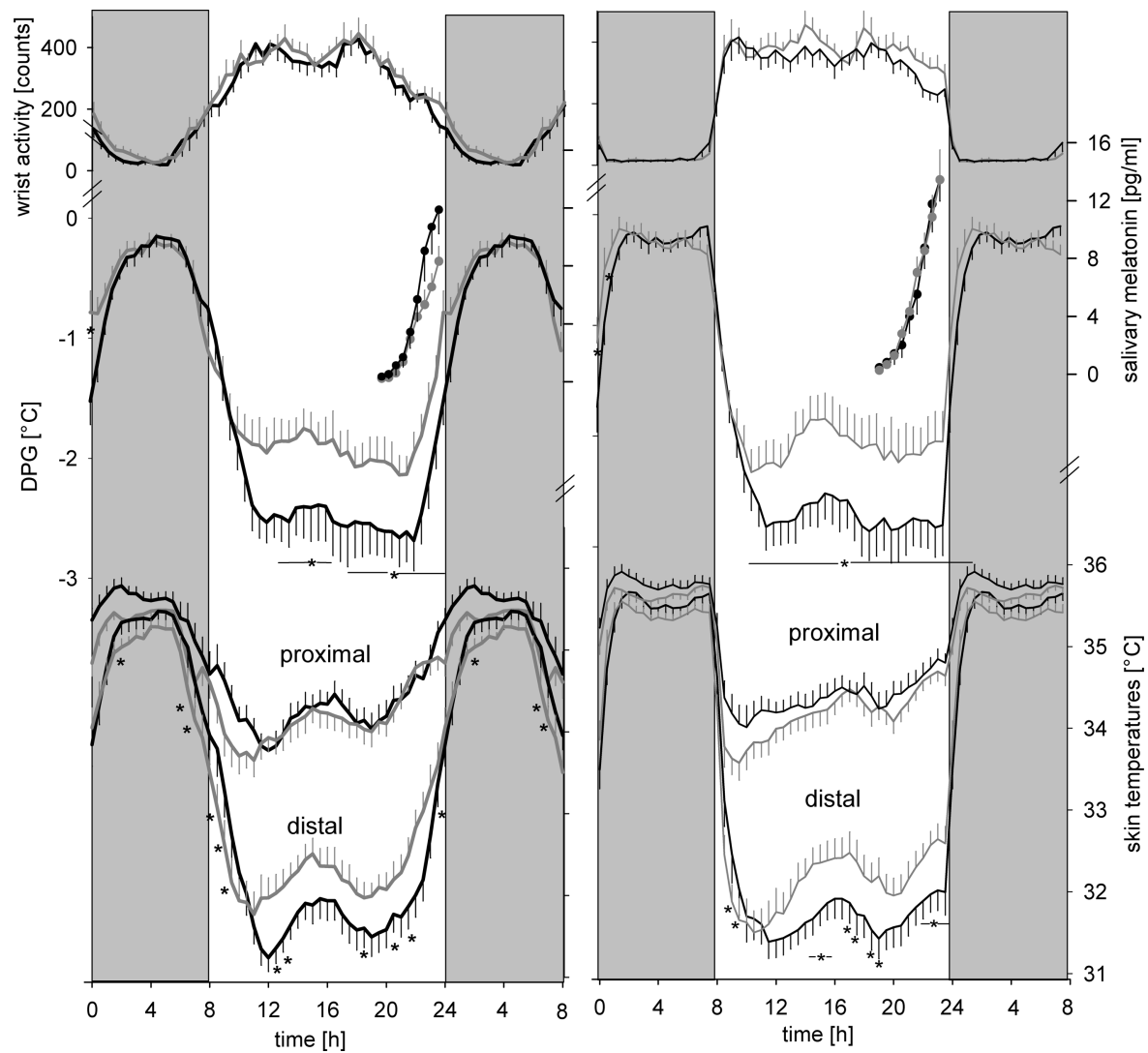

FIGURE 1 Raw and percentilized skin temperature data. From top to bottom: Diurnal pattern of wrist activity, DPG (distal-proximal skin temperature gradient), and distal and proximal skin temperatures under ambulatory conditions (mean \pm SEM; a weekly mean for each subject was calculated before averaging). Additionally, DLMO curves are inserted below the activity pattern (mean \pm SEM). Saliva samples were collected at 30-min intervals under dim light conditions at home. Grey bars indicate mean phase position of the sleep episode. Black curves: WVD $(\mathrm{N}=20)$; grey curves: $\mathrm{CON}(\mathrm{N}=$ 21). Data of the sleep episode are double plotted. Left panel represents raw data (30-min bins). In order to highlight differences between WVD and CON with respect to the sleep-wake cycle, data were percentilized separately for the sleep episode $(=100 \%)$ and for the wake phase $(=100 \%)$ (right panel). Black lines: WVD, $\mathrm{N}=19$; grey lines: $\mathrm{CON}, \mathrm{N}=19$.

.0001). During the light phase, both WVD and CON show two maxima of wrist activity, with a small afternoon trough around 15:00 h and a broad trough with minimal activity around 04:00 h.

\section{Daily Time Course of Sleep-Wake Cycle (Adjusted Data)}

Figures 1 and 2 (right panel) show the mean daily time course of skin temperatures and wrist activity adjusted to each individual sleep-wake cycle. Table 3 summarizes the results of the repeated-measures ANOVA for all variables. All skin temperatures decrease rapidly in the early 


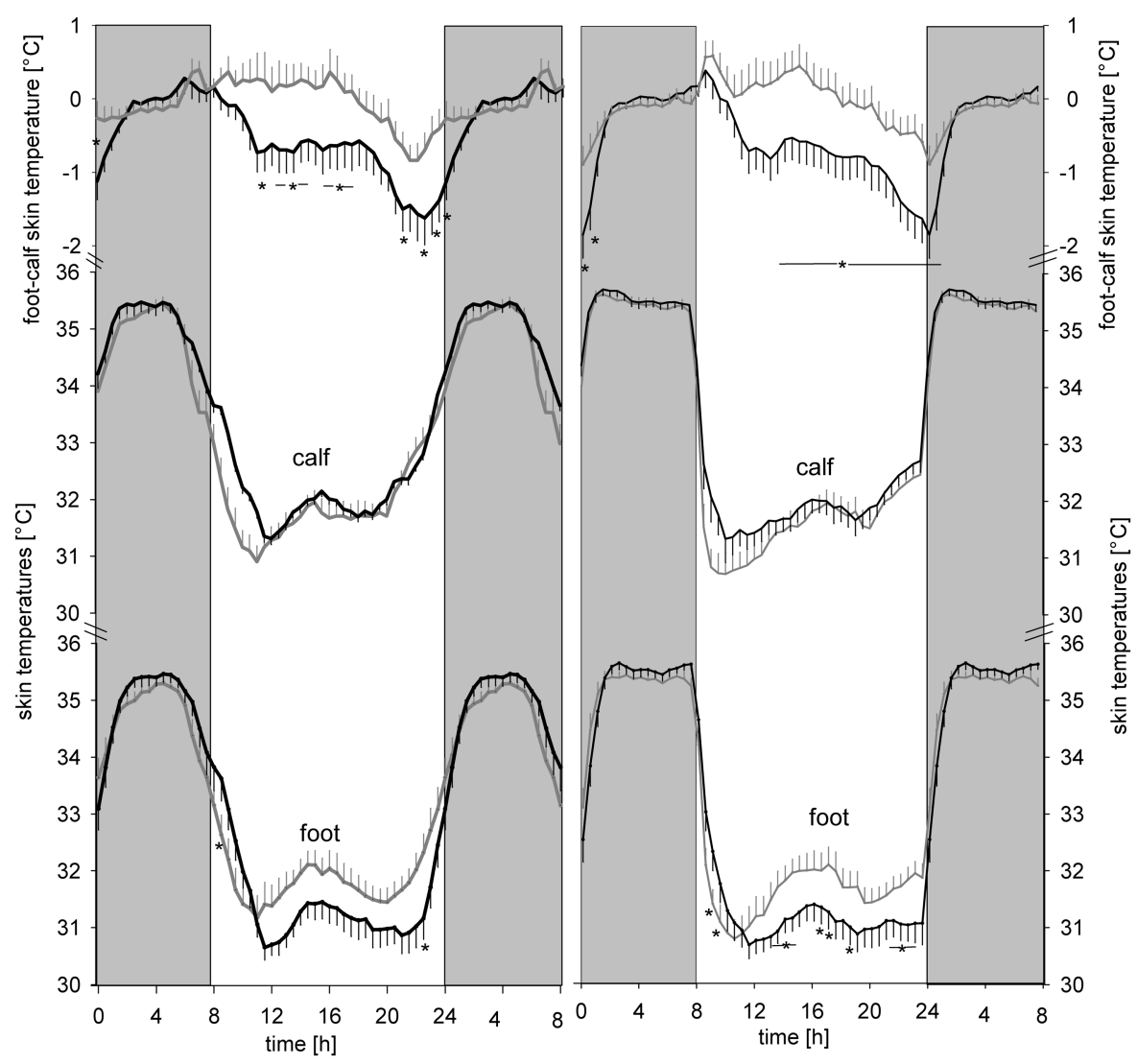

FIGURE 2 Raw and percentilized skin temperature data for lower extremities. Ambulatory data (mean values \pm SEM) of foot, calf, and foot-calf skin temperature gradient. For further descriptions see legend to Figure 1.

TABLE 2 ANOVA for wrist activity and skin temperatures adjusted to clock time

\begin{tabular}{lllllll}
\hline Variable & \multicolumn{2}{c}{ Group } & \multicolumn{2}{c}{ Time } & \multicolumn{2}{c}{ Time $\times$ Group } \\
\hline Foot & $F_{1,39}=0.84$ & $p=.360$ & $F_{47,1833}=86.60$ & $p<.001^{*}$ & $F_{47,1833}=2.82$ & $p=.016^{*}$ \\
Calf & $F_{1,39}=2.44$ & $p=.120$ & $F_{47,1833}=95.17$ & $p<.001^{*}$ & $F_{47,1833}=1.93$ & $p=.160$ \\
Distal & $F_{1,39}=0.87$ & $p=.370$ & $F_{47,1833}=108.31$ & $p<.001^{*}$ & $F_{47,1833}=2.74$ & $p=.019^{*}$ \\
Proximal & $F_{1,39}=1.38$ & $p=.250$ & $F_{47,1833}=43.73$ & $p<.001^{*}$ & $F_{47,1833}=1.17$ & $p=.320$ \\
Foot-calf & $F_{1,39}=5.40$ & $p=.025^{*}$ & $F_{47,1833}=8.96$ & $p<.001^{*}$ & $F_{47,1833}=2.42$ & $p=.027^{*}$ \\
DPG & $F_{1,39}=4.51$ & $p=.040^{*}$ & $F_{47,1833}=61.77$ & $p<.001^{*}$ & $F_{47,1833}=2.18$ & $p=.044^{*}$ \\
Wrist-activity & $F_{1,36}=0.73$ & $p=.400$ & $F_{47,1692}=48.65$ & $p<.001^{*}$ & $F_{47,1692}=0.43$ & $p=.930$ \\
\hline
\end{tabular}

Note. Two-way repeated-measures ANOVAs (rANOVAs) with factor Group (CON versus WVD) and Time $(48 \times 30$-min blocks) for the thermoregulatory variables and the wrist-activity adjusted to clock time. $\mathrm{DPG}=$ distal-proximal skin temperature gradient; feet-calf=feet-calf skin temperature gradient; ${ }^{*} p<.05$ (H-F corrected). 
TABLE 3 ANOVA for wrist activity and skin temperatures adjusted to sleep-wake cycle

\begin{tabular}{lllllll}
\hline Variable & \multicolumn{2}{c}{ Group } & \multicolumn{2}{c}{ Time } & \multicolumn{2}{c}{ Time $\times$ Group } \\
\hline Foot & $F_{1,39}=1.14$ & $p=.292$ & $F_{47,1833}=175.52$ & $p<.001^{*}$ & $F_{47,1833}=3.16$ & $p=.004^{*}$ \\
Calf & $F_{1,39}=1.99$ & $p=.170$ & $F_{47,1833}=215.69$ & $p<.001^{*}$ & $F_{47,1833}=1.37$ & $p=.230$ \\
Distal & $F_{1,39}=1.18$ & $p=.284$ & $F_{47,1833}=243.53$ & $p<.001^{*}$ & $F_{47,1833}=3.26$ & $p=.003^{*}$ \\
Proximal & $F_{1,39}=1.62$ & $p=.210$ & $F_{47,1833}=98.47$ & $p<.001^{*}$ & $F_{47,1833}=0.87$ & $p=.550$ \\
Foot-calf & $F_{1,39}=5.60$ & $p=.230$ & $F_{47,1833}=9.87$ & $p<.001^{*}$ & $F_{47,1833}=2.98$ & $p=.008^{*}$ \\
DPG & $F_{1,39}=5.06$ & $p=.030^{*}$ & $F_{47,1833}=105.72$ & $p<.001^{*}$ & $F_{47,1833}=2.98$ & $p=.009^{*}$ \\
Wrist-activity & $F_{1,36}=.64$ & $p=.430$ & $F_{47,1692}=165.36$ & $p<.001^{*}$ & $F_{47,1692}=1.10$ & $p=.360$ \\
\hline
\end{tabular}

Two-way repeated-measures ANOVAs (rANOVAs) with factor Group (CON versus WVD) and Time $(48 \times 30$-min blocks) for the thermoregulatory variables and the wrist-activity adjusted to the sleep-wake cycle (percentilized data). DPG=distal-proximal skin temperature gradient; foot-calf=footcalf skin temperature gradient; ${ }^{*} p<.05$ (H-F corrected).

morning after lights-on and, conversely, rise rapidly after lights-off. Around midday, WVD and CON differ significantly in distal, foot, footcalf, and DPG skin temperatures (significant interaction: Time $\times$ Group; significant differences between WVD and CON at certain time points are indicated by ${ }^{*} p<.05$. Proximal skin temperatures did not differ significantly between WVD and CON (middle panel). All skin temperature measures show maximal and minimal values during the night and light phase, respectively.

Actimetry revealed normally entrained sleep-wake rhythms with minimum movement during the sleep episode when skin temperatures exhibit maximal values. Two peaks of daytime activity occurred, one at midday and another in the evening. The time course of wrist activity was not significantly different in WVD compared to CON, and, in addition, the amplitude of daytime to nighttime activity in the percentilized data did not reveal any difference between the two groups $(314.3 \pm 160.4$ versus $329.6 \pm 160.4 ; F(1,36)=0.09, p=.773)$. The relative amplitude (RA) calculated from the $10 \mathrm{~h}$ of highest and $5 \mathrm{~h}$ of least activity, as a circadian parameter, tended to be higher in WVD compared to CON (RA; $0.88 \pm 0.08$ versus $0.92 \pm 0.04 ; F(1,36)=3.3, p=.078)$.

\section{Phase Relationship Analyses}

The intraindividual phase relationships between foot skin temperatures and wrist activity were quantified by cross-correlation analysis (Figure 3, upper panel). In comparison to CON, WVD show an asymmetric cross-correlation curve, with distinct differences between lags -8 and $-1 \mathrm{~h}$. Individual extracted times of minimum $r$ values in $\mathrm{CON}$ reveal a significant phase advance of foot skin temperatures with respect to wrist activity $(p<.05$; phase of wrist activity was set as 0$)$. Compared with $\mathrm{CON}$, the phase position of WVD (wrist-foot correlation) is significantly 


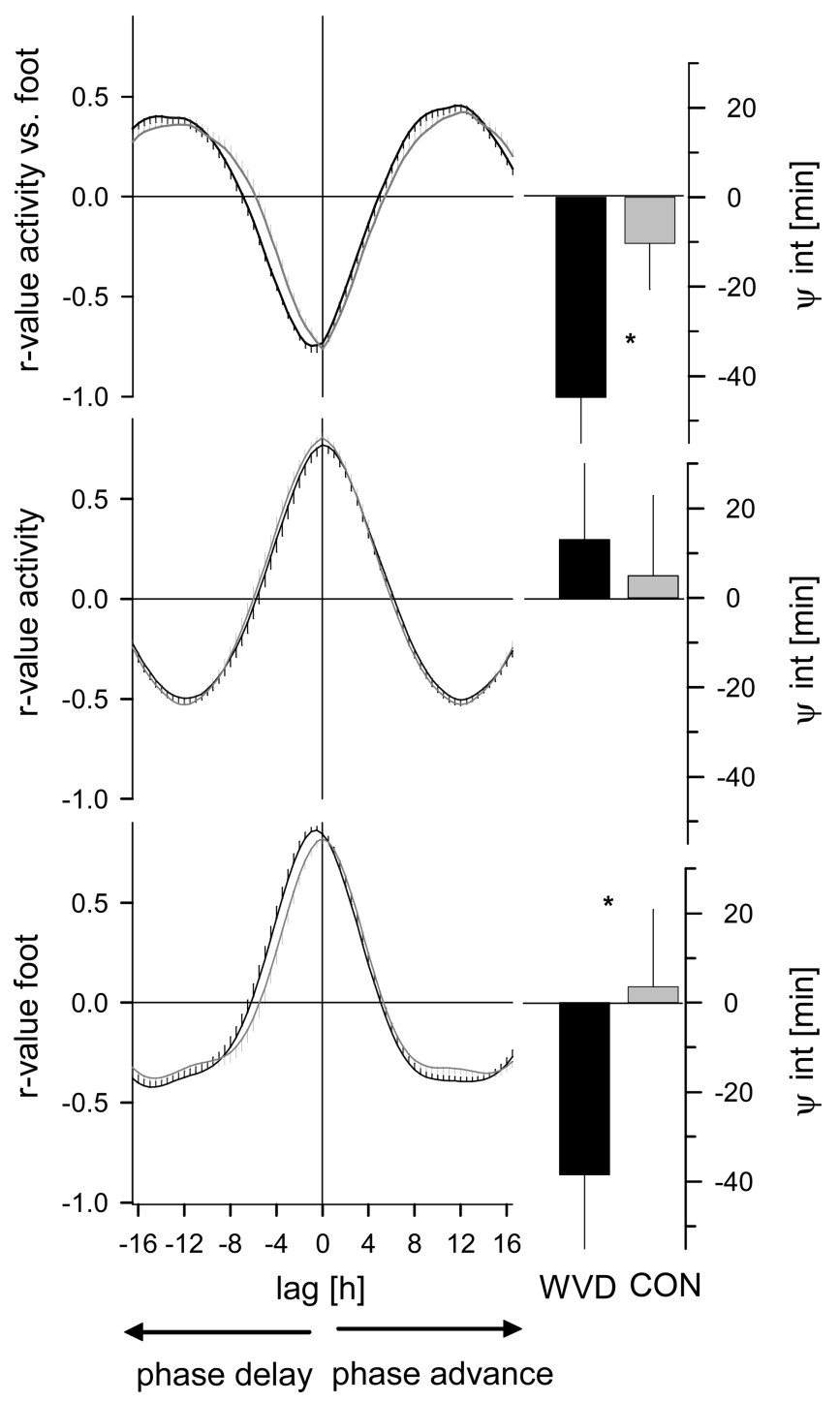

FIGURE 3 Cross-correlation analyses of wrist and temperature data. Left panel: Data are shown in mean values \pm SEM; WVD: black lines; CON: grey lines. Top left panel: Mean of intra-individual foot skin temperature versus wrist activity cross-correlations (WVD: $N=20$; CON: $N=18$ ). Middle left panel: Mean cross-correlation curves of wrist activity versus mean wrist activity of CON. Data of all individuals (WVD: $\mathrm{N}=20$; $\mathrm{CON}$ : $\mathrm{N}=18$ ) were cross-correlated with the mean wrist activity pattern of CON $(\mathrm{N}=18)$. Bottom left panel: Mean cross-correlation curves of foot skin temperature versus mean foot skin temperature of CON. Data of all individuals (WVD: $N=20$; $C O N$ : $N=21$ ) were cross-correlated with the mean foot skin temperature pattern of CON $(\mathrm{N}=21)$. Right panel: Phase angle differences between CON (grey bars) and WVD (black bars); mean values \pm SEM. Lags were extracted from maximum or minimum values of individual cross-correlation graphs. $p$ values were calculated with the Mann-Whitney $U$ test. 0 lag $=$ in phase; +lag $=$ phase advance, - lag $=$ phase delay. Top right panel: wrist activity versus foot skin temperature cross-correlations; 0 lag $=$ in phase with wrist-activity (wrist activity versus foot: ${ }^{*} p<.01$ ). Middle right panel: wrist activity versus mean wrist activity of $\mathrm{CON}$; $0 \mathrm{lag}=$ in phase with wrist-activity of $\mathrm{CON}$ (wrist activity versus mean wrist activity of CON: ns). Bottom right panel: foot skin temperatures versus mean foot skin temperature of $\mathrm{CON} ; 0 \mathrm{lag}=$ in phase with mean foot skin temperatures of $\left.\mathrm{CON}:{ }^{*} p<.05\right)$. 
phase delayed by $35 \mathrm{~min}(p<.01)$. The cross-correlation curves of wrist activity are similar in WVD and CON (Figure 3, middle panel), and the extracted phases do not differ statistically. The cross-correlation curve of foot skin temperatures of $\mathrm{WVD}$ and $\mathrm{CON}$ reveal clear differences (Figure 3, lower panel). Compared with CON, the time course of foot skin temperatures in WVD is significantly phase-delayed by $42 \mathrm{~min}(p<$ $.05)$. Taken together, the daily time course of foot skin temperatures in WVD is significantly phase delayed by $35-42 \mathrm{~min}$ with respect to wrist activity and CON.

\section{Subjective Measures}

Sleepiness and concentration measured by VAS exhibit a significant interaction (Time $\times$ Group, $p<.05$; Figure 4 ), showing that WVD are less tired and more concentrated than CON before lights-off. Morning ratings show an inverse pattern, leading to lower daily fluctuations in WVD.

WVD showed higher ratings of cold sensations than CON for all skin regions (Figure 4; main effect Group, $p<.02$ ). Additionally, WVD exhibited a stronger sensation of cold feet than CON before sleep (Time $\times$ Group, $p<.02$ ).

WVD did not differ from CON in subjective ratings of mood, tension, and feelings of hunger. All subjects were more hungry, more relaxed, and in better mood in the evening than morning.

\section{Salivary Melatonin Concentrations in the Evening and DLMO}

The time course of salivary melatonin concentrations is presented in Figure 1 for clock time (left panel, 19:00-23:00 h) and lights-off (sleepwake cycle)_adjusted data (right panel). Statistical comparisons were performed by two-way, repeated-measures ANOVA. Neither clock time nor sleep-wake-adjusted time courses reveal significant differences between WVD and CON. Clock-time-adjusted data for: Group, WVD versus CON, $F(1,37)=2.00, p=.16$, n.s.; Time, 9 timepoints, $F(8,296)=$ $55.58, p<.0001$; Time $\times$ Group, $F(8,296)=2.83, p=.067 ; p<.05(\mathrm{H}-\mathrm{F}$ corrected). Lights-off adjusted data for: Group, WVD versus CON, $F(1$, $37)=0.01, p=.92$, n.s.; Time, 9 timepoints, $F(8,296)=66.47, p<.0001$; Time $\times$ Group, $F(8,296)=0.34, p=.95$, n.s.

Statistical analysis of the DLMO times reveals no differences between the groups. Clock-time adjusted data: WVD versus CON, 21.54 \pm 0.26 versus $21.47 \pm 0.28 \mathrm{~h}, F(1,37)=0.04, p=.852$, n.s.; lights-off adjusted data: WVD versus CON, $2.14 \pm 0.21$ versus $1.83 \pm 0.23 \mathrm{~h}$ before lightsoff, $F(1,37)=0.99, p=.326$, n.s.

DLMO correlated significantly with the phase of activity $(r=.66, \mathrm{n}=$ $38, p<.0001)$, feet skin temperature $(r=.66, \mathrm{n}=41, p<.0001)$, and 


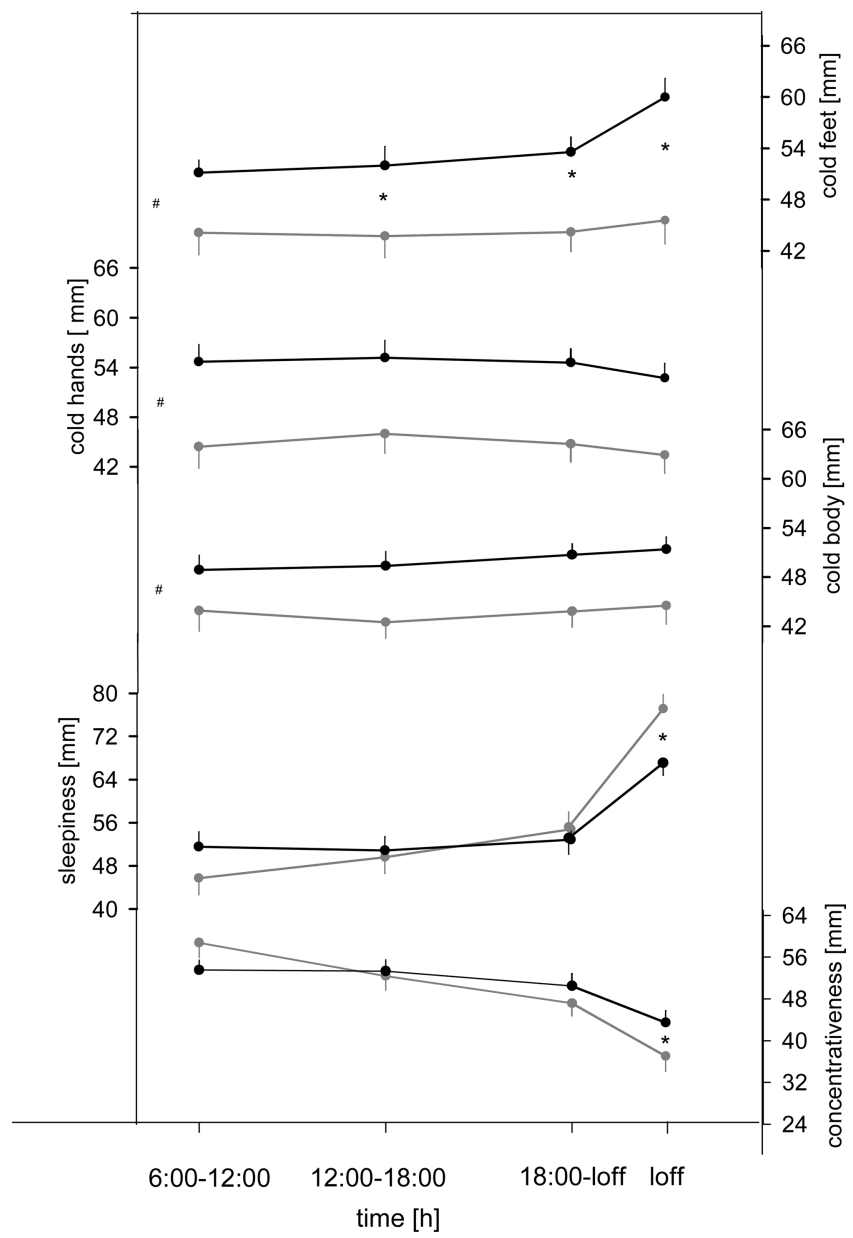

FIGURE 4 Subjective data. Subjective VAS data (worse = higher values) of thermal discomfort (cold feet, cold hands, and cold body), sleepiness, and concentration (WVD: $\mathrm{N}=20$, black curve; CON: $\mathrm{N}=21$, grey curve). Data (mean \pm SEM) are displayed in time spans (06:00-12:00 h; 12:00-18:00 h; 18:00 h, and lights-off [loff]). Significant interaction: time $\times \mathrm{WVD} / \mathrm{CON},{ }^{*} p<.05$; significant main effect: $\mathrm{WVD} / \mathrm{CON},{ }^{\#} p<.05$.

sleep midpoint $(r=0.7, \mathrm{n}=41, p<.0001)$. Taken together, irrespective of whether the data are adjusted to clock time or the individual sleepwake cycle, the timing of the rise in salivary melatonin concentration (DLMO) did not differ statistically between WVD and CON.

\section{DISCUSSION}

This ambulatory study of WVD revealed two main findings. First, their thermoregulatory systems exhibited a different circadian phase of entrainment than $\mathrm{CON}$, in that distal (but not proximal) skin temperatures were phase delayed with respect to the habitual sleep-wake cycle by 
about 40 min. Second, WVD, who complain of thermal discomfort with cold extremities, do, in fact, exhibit objectively measured cooler skin temperatures than $\mathrm{CON}$, selectively at the extremities, and this occurs throughout the day, but not during the sleep episode. The two subject groups were selected with respect to VD and DIS. Therefore, further studies including all four combinations of $\mathrm{VD}$ and DIS are required to elucidate whether it is VD and/or DIS that is essential for the phase delay of distal skin temperature in WVD.

The discussion of the findings is structured into differences in phase of entrainment and vascular regulation. In the last part, we try to elucidate how the thermoregulatory system interacts with the circadian system.

\section{Differences in Phase of Entrainment}

The starting point of the present investigation was our recent constant-routine (CR) laboratory study showing parallel phase delays of about $1 \mathrm{~h}$ in all measured circadian variables (CBT, skin temperatures, DPG, salivary melatonin, and subjective ratings of sleepiness) in WVD with respect to CON (Kräuchi, 2007). Similar to the present study, sleep times (time of lights-off and lights-on) did not differ between the two groups. However, in contrast to the CR study, under ambulatory conditions only the diurnal pattern of DPG was phase delayed, whereas DLMO did not differ.

How can this discrepancy between CR and ambulatory conditions be explained? The differences between these two protocols lay not only in differences in body position, physical exercise, food intake patterns, and increasing sleepiness (masking effects), but most importantly also in the lighting conditions. It is well known that overt circadian rhythms, including that of melatonin, are generated by the circadian pacemaker system localized in the SCN, which is efficiently entrained by light (for review see Dijk \& von Schantz, 2005). Subjects in a CR experience a $<8$-lux environment that allows the measurement of unmasked circadian phase and amplitude. In the real world, they are exposed to much brighter light intensities at variable times of the day, depending on their work and free-time habits. Thus, the masking and entraining effects of light may be responsible for the similar phases of WVD and CON in DLMO, wrist activity, and proximal skin temperature under ambulatory conditions. Only the phase delay in distal skin temperature and the diurnal vasoconstriction remained also during ambulatory conditions. Thus, we can link the delayed sleep onset latency to the delayed onset of vasodilatation in the evening and not to a phase shift of the circadian clock.

Many studies have shown that the distal heat loss regulatory system does not act unidirectionally on sleep initiation. All kinds of relaxation behavior prior to sleep, whether muscular or psychological in nature (e.g., 
lying down, sleep initiation rituals), trigger a pronounced increase in distal vasodilatation. All temperatures increase rapidly after lights-off, whether under ambulatory or CR conditions (Kräuchi et al., 1999; Lack \& Gradisar, 2002). Such a feedback regulatory system can be used therapeutically for sleep onset disturbances, e.g., by applying bed socks, autosuggestion of warmth, autogenic training, and yoga (Kräuchi et al., 1997, 1999).

In the present study, we also found that WVD reported lower sleep quality and more awakenings during sleep than CON, which could have influenced alertness the following morning. Indeed, WVD showed a tendency to stay longer in bed and to be sleepier in the morning, both indicative of a more pronounced sleep inertia process (Kräuchi et al., 2006). This finding fits together with the longer duration of the distal vasoconstriction process in the morning in WVD; the disappearance of sleep inertia has been shown to correlate with the rate of distal vasoconstriction (Kräuchi et al., 2004). WVD rated not only more sleepiness in the morning but also less sleepiness in the evening (Figure 4), which might indicate a phase delay of the circadian sleep propensity rhythm as previously found in a CR study (Vollenweider et al., 2008). This interpretation is in accord with previous findings showing a close relationship between the circadian rhythm of sleepiness and distal vasodilatation (Kräuchi et al., 2006; Lack et al., 2008).

\section{Differences in Vascular Regulation}

Our study revealed interesting findings with respect to circadian thermoregulation. The daily subjective ratings of cold sensation throughout the day confirmed the global rating of the screening instrument. WVD had higher ratings of cold sensation, most pronounced in distal skin regions, especially their feet, before lights-off. This finding provides information about the status of the thermoregulatory systems of WVD and CON. All subjects usually try to reach a state of thermal comfort, which requires a thermoregulatory system in equilibrium. Therefore, recognition of thermal discomfort is claimed to be an indication of a deviation in the subjects' thermoregulatory "set point" (Cabanac, 1969). WVD not only feel that they have cooler distal skin regions while awake, but they, indeed, do have cooler measured distal skin temperatures than CON. Interestingly, both groups had similar proximal skin temperatures, leading, therefore, to a clear distinction between the two groups in DPG during the wake phase. The skin temperatures of the calf and foot, and their difference, may provide an additional insight into thermoregulatory heat loss mechanisms via the lower legs. The calf skin temperature exhibits its lowest values in the morning, increasing thereafter, with the exception of a small trough in the afternoon, to high values before 
lights-off. Even though the spatial distance between the temperature probes of the calf and foot was only about $25 \mathrm{~cm}$, the temperature difference between them during the wake phase was quite large (average: $0.73^{\circ} \mathrm{C}$ in $\left.\mathrm{WVD}\right)$. This indicates that effective thermoregulatory mechanisms have to be activated to induce such a large gradient in skin temperatures within this short distance, e.g., closing of AVAs and countercurrent back stream of blood via inner veins. Within this context, it is noteworthy that the parallel bimodal pattern of proximal and distal skin temperatures occurring during the daytime, with maximum values around 16:00 h, are probably not of thermoregulatory origin and suggest a behavioral one (e.g., food intake). Such a bimodal pattern of ambulatory distal skin temperature has been previously documented (Sarabia et al., 2008).

Remarkable is that WVD exhibit a slightly higher life stress level than $\mathrm{CON}$, and a higher propensity to react with cold hands in stressful situations. Further support for a higher stress sensitivity in WVD comes from other analyses where we have shown that WVD in comparison to CON exhibit reduced parasympathetic activity and increased sympathovagal balance, as derived from heart rate variation (HRV) spectral analyses (Anders et al., 2010), and a higher anger-turned-inwards level (Von Arb et al., 2009), which in turn could be responsible for the increased sympathovagal balance and distal vasoconstriction.

Analysis of percentilized data (Figures 1 and 2, right panel) illustrates how vasodilatation and vasoconstriction are tightly linked to the sleepwake cycle after lights-off and lights-on. In both WVD and CON, distal skin temperatures reached their minimum and maximum levels later in the morning and night, respectively, than proximal skin temperatures, confirming the findings under controlled CR conditions (Kräuchi et al., 2006). As a consequence, distal skin regions exhibited a $2-3^{\circ} \mathrm{C}$ lower diurnal temperature level than proximal skin regions. The thermoregulatory difference between WVD and CON lies rather in the different temperature levels, i.e., lower DPG levels during the diurnal phase in WVD, than in the kinetics of the distal thermoregulatory heat loss system.

In conclusion, WVD live with a larger thermoregulatory "shell" during the wake phase resulting in increased body heat retention, a state occurring when the thermoregulatory "set levels" are not reached. Increased distal vasoconstriction may be caused by their documented elevated life stress level. Having vasoconstricted distal skin regions before lights-off are known to prolong sleep onset latency. The demonstrated phase delayed distal skin temperature rhythm with respect to the sleepwake cycle in WVD could also be caused by lower diurnal distal skin temperatures. It should be noted that we studied a healthy population whose delayed sleep onset could be linked to the above thermoregulatory disturbance, while maintaining similar circadian phase as controls. Sleeponset disturbances in the clinic, however, have a variety of origins; for 
example, some could be related to thermoregulation and stress and others to delayed internal clock time. What we have shown is, in this model population, that the degree of vascular dysregulation of a subject may represent a crucial thermophysiological property that is necessary for initiating sleep.

\section{ACKNOWLEDGMENTS}

This work was supported by the Swiss National Science Foundation Grant SNF 3100A0-102182/1 (to K. Kräuchi), and the SchwickertStiftung. The authors are grateful to Anna Wirz-Justice for her helpful comments on the manuscript. The authors are grateful to Anna WirzJustice for her helpful comments on the manuscript and Sarah Chellapa for her linguistic corrections. Furthermore, the authors acknowledge Claudia Renz, Marie France Dattler, and Giovanni Balestrieri for their technical aid.

Declaration of Interest: The authors report no conflicts of interest. The authors alone are responsible for the content and writing of the paper.

\section{REFERENCES}

Anders D, Vollenweider S, Cann J, Hofstetter M, Flammer J, Orgül S, Kräuchi K. (2010). Heart rate variability in women during 40 hour prolonged wakefulness. Chronobiol. Int. 27:1609-1628.

Aschoff J. (1971). Temperaturregulation. In Gauer OH, Kramer K, Jung R (eds). Energiehaushalt und Temperaturregulation. Physiologie des Menschen. München: Urban und Schwarzenberg, 43-112.

Aschoff J, Heise A. (1972). Thermal conductance in man: its dependence on time of day and of ambient temperature. In Itoh S, Ogata K, Yoshimura H (eds) Advances in climatic physiology. Tokyo: Igako Shoin, 334-348.

Cabanac M. (1969). Plaisir ou deplaisir de la sensation thermique et homeothermie. Physiol. Behav. 4:359-364.

Campbell SS, Broughton RJ. (1994). Rapid decline in body temperature before sleep: fluffing the physiological pillow? Chronobiol. Int. 11:126-131.

Curran-Everett D. (2000). Multiple comparisons: philosophies and illustrations. Am. J. Physiol. Regul. Integr. Comp. Physiol .279:R1-R8.

Dijk D, von Schantz M. (2005). Timing and consolidation of human sleep, wakefulness, and performance by a symphony of oscillators. J. Biol. Rhythms 20:279-290.

Flammer J, Pache M, Resink T. (2001). Vasospasm, its role in the pathogenesis of diseases with particular reference to the eye. Prog. Retin. Eye Res. 20:319-349.

Hales JRS. (1985). Skin arteriovenous anastomoses, their control and role in thermoregulation. In Johansen K, Burggren WW (eds). Cardiovascular shunts. Alfred Benzon Symposium, Copenhagen: Munksgaard, 433-451.

House JR, Tipton MJ. (2002). Using skin temperature gradients or skin heat flux measurements to determine thresholds of vasoconstriction and vasodilatation. Eur. J. Appl. Physiol. 88:141-145.

Kräuchi K. (2007). The thermophysiological cascade leading to sleep initiation in relation to phase of entrainment. Sleep Med. Rev. 11:439-451.

Kräuchi K, Wirz-Justice A. (1994). Circadian rhythm of heat production, heart rate, and skin and core temperature under unmasking conditions in men. Am. J. Physiol. 267:R819-R829.

Kräuchi K, Cajochen C,Wirz-Justice A. (1997). A relationship between heat loss and sleepiness: effects of postural change and melatonin administration. J. Appl. Physiol. 83:134-139. 
Kräuchi K, Cajochen C, Werth E, Wirz-Justice A. (1999). Warm feet promote the rapid onset of sleep. Nature 401:36-37.

Kräuchi K, Cajochen C, Werth E, Wirz-Justice A. (2000). Functional link between distal vasodilation and sleep-onset latency? Am. J. Physiol. Regul. Integr. Comp. Physiol. 278:R741-R748.

Kräuchi K, Cajochen C, Wirz-Justice A. (2004). Waking up properly: is there a role of thermoregulation in sleep inertia? J. Sleep Res. 13:121-127.

Kräuchi K, Knoblauch V, Wirz-Justice A, Cajochen C. (2006). Challenging the sleep homeostat does not influence the thermoregulatory system in men: evidence from a nap vs. sleep-deprivation study. Am. J. Physiol. Regul. Integr. Comp. Physiol. 290:R1052-R1061.

Kräuchi K, Fontana Gasio P, Vollenweider S, Von Arb M, Dubler B, Orgül S, Flammer J, Stutz E. (2008). Cold extremities and difficulties initiating sleep: evidence of co-morbidity from a random sample of a Swiss urban population. J. Sleep Res. 17:420-426.

Lack L, Gradisar M. (2002). Acute finger temperature changes preceding sleep onsets over a 45-h period. J. Sleep Res. 11:275-282.

Lack L, Gradisar M, Van Someren EJW, Wright HR, Lushington K. (2008). The relationship between insomnia and body temperatures. Sleep Med. Rev. 12:307-317.

Pandi-Perumal SR, Smits M, Spence W, Srinivasan V, Cardinali DP, Lowe AD, Kayumov L. (2007). Dim light melatonin onset (DLMO): a tool for the analysis of circadian phase in human sleep and chronobiological disorders. Prog. Neuropsychopharmacol. Biol. Psychiatry 31:1-11.

Portaluppi F, Touitou Y, Smolensky M. (2008). Ethical and methodological standards for laboratory and medical biological rhythm research. Chronobiol. Int. 25:999-1016.

Roenneberg T, Daan S, Merrow M. (2003a). The art of entrainment. J. Biol. Rhythms 18:183-194.

Roenneberg T, Wirz-Justice A, Merrow M. (2003b). Life between clocks: daily temporal patterns of human chronotypes. J. Biol. Rhythms 18:80-90.

Rubinstein E, Sessler D. (1990). Skin-surface temperature gradients correlate with fingertip blood flow in humans. Anesthesiology 73:541-545.

Sarabia J, Rol M, Mendiola P, Madrid J. (2008). Circadian rhythm of wrist temperature in normalliving subjects A candidate of new index of the circadian system. Physiol. Behav .95:570-580.

Severens NM, van Marken Lichtenbelt WD, Frijns AJ, Kingma BR, de Mol B A, van Steenhoven A A. (2010). Measurement of model coefficients of skin sympathetic vasoconstriction. Physiol. Meas. 31:77-93.

Smith AD, Crabtree DR, Bilzon JL, Walsh NP. (2010). The validity of wireless iButtons and thermistors for human skin temperature measurement. Physiol. Meas. 31:95-114.

Van Marken Lichtenbelt WD, Daanen HA, Wouters L, Fronczek R, Raymann RJ, Severens NM, Van Someren EJ. (2006). Evaluation of wireless determination of skin temperature using iButtons. Physiol. Behav. 88:489-497.

Van Someren EJ, Swaab DF, Colenda CC, Cohen W, McCall WV, Rosenquist PB. (1999). Bright light therapy: improved sensitivity to its effects on rest-activity rhythms in Alzheimer patients by application of nonparametric methods. Chronobiol. Int. 16:505-518.

Vollenweider S, Wirz-Justice A, Flammer J, Orgül S, Kräuchi K. (2008). Chronobiological characterization of women with primary vasospastic syndrome: body heat loss capacity in relation to sleep initiation and phase of entrainment. Am. J. Physiol. Regul. Integr. Comp. Physiol. 294:R630-R638.

Von Arb M, Gompper B, Meyer AH, Stutz EZ, Orgül S, Flammer J, Kräuchi K. (2009). Relationship between gender role, anger expression, thermal discomfort and sleep onset latency in women. Biopsychosoc. Med. 3:1-7.

Weber J, Schwander J, Unger I, Meier D. (1997). A direct ultrasensitive RIA for the determination of melatonin in human saliva: comparison with serum levels. Sleep Res. 26:A112-A113.

Werner J. (2010). System properties, feedback control and effector coordination of human temperature regulation. Eur. J. Appl. Physiol. 109:13-25.

Wever R. (1979). The circadian system of man-results of experiments under temporal isolation. New York: Springer Verlag. 